\title{
HYDROLYTIC STABILITY OF DILTIAZEM IN THE PRESENCE OF CHOSEN POLYMERS - POLOXAMER 407 AND AMMONIO METACRYLATE COPOLYMER TYPE B (EUDRAGIT RS): A PRELIMINARY STUDY

\author{
Nadezhda Ivanova ${ }^{1}$, Stanila Stoeva ${ }^{2}$, Iliyan Kolev ${ }^{3}$ \\ ${ }^{1}$ Department of Pharmaceutical Technologies, Faculty of Pharmacy, \\ Medical University of Varna \\ ${ }^{2}$ Department of Pharmacology, Toxicology and Pharmacotherapy, Faculty of Pharmacy, \\ Medical University of Varna \\ ${ }^{3}$ Department of Pharmaceutical Chemistry, Faculty of Pharmacy, \\ Medical University of Varna
}

\begin{abstract}
INTRODUCTION: The hydrolytic stability of the calcium channel blocker representative diltiazem hydrochloride in the presence of different polymeric entourage was quantitatively assessed using HPLC.

AIM: The experiment was performed in order to establish drug-excipients compatibility in Poloxamer 407 hydrogel formulations, where the drug was introduced either by a direct (conventional) approach or in the form of Eudragit RS microsponge-type particles with sustained drug release.

MATERIALS AND METHODS: Samples of conventional diltiazem 2\% hydrogel and microsponge-enriched diltiazem $2 \%$ hydrogel were stored at $25 \pm 2^{\circ} \mathrm{C}$ for 10 months, protected from light. Analysis of the chemical decomposition rate of diltiazem hydrochloride to its desacetyl degradant - O-desacetyl-diltiazem hydrochloride - was performed in the period between $4^{\text {th }}$ and $10^{\text {th }}$ month of storage.

RESULTS AND DISCUSSION: It was hypothesized that a polymeric "shield" of Eudragit RS in the composition of diltiazem-loaded porous microspheres and/or the enhanced viscosity achieved by the addition of high-molecular gelling agent Poloxamer 407 to an aqueous drug dispersion will benefit the drug's stability. Instead, a negative effect on the hydrolysis rate was found to be dominant for both polymers, likely due to an "unfavorable" shift in the dielectric conductivity of the media and/or suspected catalytic effect of quaternary ammonium groups of Eudragit $R S$.
\end{abstract}

Address for correspondence:

Nadezhda Ivanova

Faculty of Pharmacy

Medical University of Varna

84 Tzar Osvoboditel Blvd

9000 Varna

e-mail: nadejda.ivanova@mu-varna.bg

Received: March 15, 2019

Accepted: June 8, 2019
CONCLUSION: These preliminary results led us to a deeper understanding of the polymeric impact on diltiazem hydrolytic behavior and a very useful foundation for a future preformulation stage in the development of diltiazem modified-release semisolid forms for the treatment of chronic anal fissure.

Keywords: diltiazem hydrolysis, diltiazem stability, drug-excipient compatibility, polymeric drug delivery, HPLC 


\section{INTRODUCTION}

The chemical stability of an active pharmaceutical ingredient is of critical value for the dosage forms endurance and shelf life. Hydrolysis, as a common process leading to drug decomposition, affects primarily ester, amide, imide, thioester, lactam, and lactone functional groups. The kinetics and mechanism of the hydrolytic process depend on intrinsic (related to the nature of the drug compounds) and external (environmental) factors. Important compound-related factors are the type of hydrolytically unstable bond (e.g., ester, amide etc.), electrophilicity of the carbonyl C-atom, substituents by and around the same, steric stabilization, potential for intramolecular catalysis, ionizability, ability of the leaving group (e.g., alcohol), polymorphism and reactive surface area (drug particle size). The most important among external factors are the temperature, $\mathrm{pH}$, moisture/water content, water mobility, ionic strength, presence of catalytically active ions and functional groups (intermolecular catalysis), dielectric constant, and type of drug dispersion (e.g., a solution or a suspension). All variables belonging to the group of environmental factors (with the exception of temperature) may be a function of the excipients used in a dosage form (1).

It was of our interest to follow the impact of two commonly used polymers in the pharmaceutical industry - Poloxamer 407 and ammonio metacrylate copolymer Type B (Eudragit RS) on the hydrolytic degradation of diltiazem hydrochloride - a vasodila-

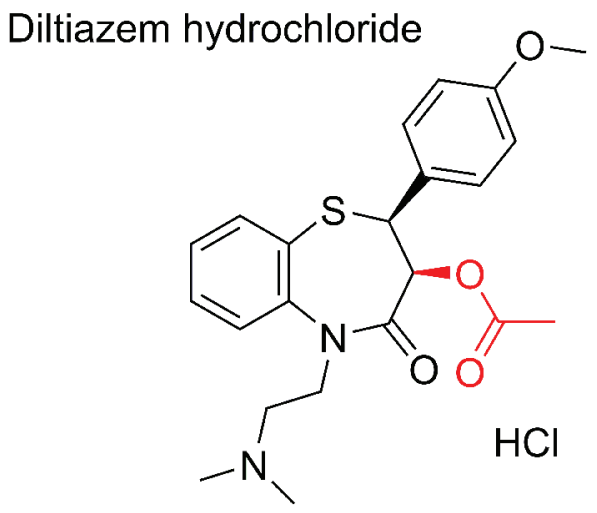

Hydrochloride of (2S,3S)-5-[2-(dimethylamino)ethyl]2-(4-methoxyphenyl)-4-oxo-2,3,4,5-tetrahydro-1,5benzothiazepin-3-yl acetate

Scheme 1. Structure of diltiazem hydrochloride tor of the calcium channel blocker group - in hydrogel dispersions.

The latter are applicable in the treatment of chronic anal fissure (CAF), but are most often prepared ex tempore, given the low stability of the compound in a hydrated environment and respective short shelf life.

Essential for the hydrolytic degradation of diltiazem is its acetyl oxide radical (2). Hydrolysis of the $\mathrm{CH}_{3} \mathrm{COO}$-radical results in the formation of equimolar amounts of $\mathrm{O}$-desacetyl diltiazem (des-diltiazem) and acetic acid (Fig. 1) (3,4). Des-diltiazem possesses only quarter to half of the pharmacological activity of the parent drug molecule $(5,6)$. The relatively high hydrolytic stability of the 7-membered lactam ring of diltiazem, on the other side, is likely due to a steric stabilization. Maximal stability and lowest rate of hydrolysis of diltiazem hydrochloride are reported at low-pH values in the range 3-5 $(1,4,7)$.

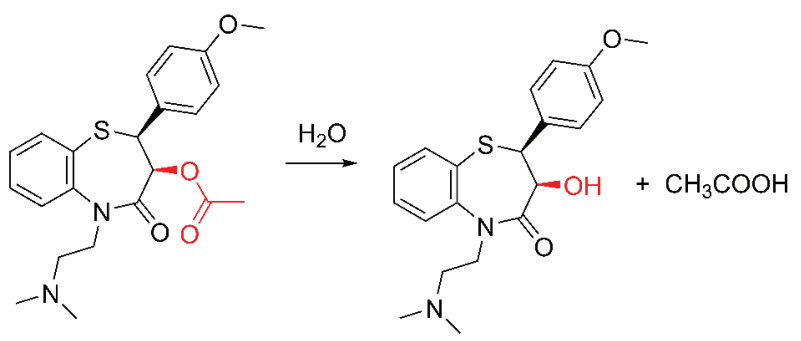

Fig. 1. Hydrolysis of diltiazem to des-diltiazem

In addition, under the influence of UV-Vis electromagnetic radiation, diltiazem is found to epimerize reversibly to its $(+)-(2 \mathrm{R}, 3 \mathrm{~S})$-trans diltiazem antipode, owing significantly lower coronaro-dilator ability $(8,9)$. The photoinstability of diltiazem should be considered when choosing a primary packaging for the substance in bulk and diltiazem containing dosage forms (1).

The high reactivity and low hydrolytic stability of diltiazem occurs both in vitro (during storage) and in vivo (after intake). The latter manifests as low resistance to esterases and other enzymes (10). Respectively, diltiazem owns a short biological half-life $\left(\mathrm{t}_{1 / 2}\right)$ and oral bioavailability $(\mathrm{F})\left(\mathrm{t}_{1 / 2} 3-4.5 \mathrm{~h} ; \mathrm{F} \approx 40 \%\right)(4-6)$. In this view, there is a pharmacokinetic prerequisite to formulate diltiazem in modified-release dosage forms. The same applies when it comes to rectal dil- 
Nadezhda Ivanova, Stanila Stoeva, Iliyan Kolev

tiazem dosage forms. In this case, the sustained drug release overcomes some drawbacks showed by the conventional topical diltiazem hydrochloride formulations for the treatment of CAF. A sustained drug delivery form of Eudragit RS microsponge-based Poloxamer diltiazem $2 \%$ hydrogels demonstrated a better drug deposition in rectal mucosa and lower absorption, thus declaring the potency to reduce anal pruritus and postural hypotension caused by fast dissolution and absorption of diltiazem hydrochloride (11).

\section{AIM}

The aim of the current survey was to assess the impact of the used polymers - Eudragit $R S$ and Poloxamer 407 - on diltiazem with respect to its hydrolytic stability.

\section{MATERIALS AND METHODS}

1. Materials - Chemicals and reagents were obtained from (as follows): diltiazem hydrochloride 99.9\% - Puho Pharmaceuticals Co. Limited, China; desacetyl diltiazem hydrochloride - LGC GmbH, Germany; ammonio methacrylate copolymer (type B) (Eudragit RS 100) Evonik Industries AG, Germany; Poloxamer 407 (Lutrol F-127) - BASF, Germany; Ethanol Absolute HPLC grade and Methanol HPLC grade - Fisher Chemical, Germany; orthophosphoric acid $>85 \%$ - Fisher Scientific, UK.

2. Preparation of diltiazem $2 \%$ gels - two types of diltiazem 2\% gels were prepared, using gel base Poloxamer 407 20\%:

1. Diltiazem 2\% Poloxamer 20\% hydrogel (PxDtz-gel), where the drug was introduced in the gel base via water solution (direct approach) and

2. Microsponge-enriched diltiazem $2 \%$ Poloxamer 20\% hydrogel (micro-Px-Dtz-gel), where the drug was introduced in the gel base via diltiazemloaded Eudragit RS 100 microsponge-type particles.

Reference for the descriptive preparation techniques (for the gels formulation and the synthesis of diltiazem-loaded microsponge-type carriers) is available in our previous works (11-13). Sample of each formula was stored at $25 \pm 2^{\circ} \mathrm{C}$ in the dark for a period of 10 months. A standard diltiazem 2\% solution in distilled water was used as reference; it was stored and tested under the same conditions.
3. Preparation of standard solutions - standard stock solutions of diltiazem and desdiltiazem were prepared in methanol at concentration of $100 \mu \mathrm{g} / \mathrm{mL}$. Dilutions were performed by mixing both stock solutions in gradient percent ratios from $0 \%$ to $100 \%$ by a step of $10 \%$. Standard calibration curves of diltiazem and des-diltiazem were built by using HPLC data.

4. Preparation of test solutions - $0.500 \mathrm{~g}$ of each test gel was dissolved in sufficient volume of methanol and further diluted up to $25.0 \mathrm{ml}$ in a volumetric flask. Proper dilution with the same solvent was carried, where it was necessary, to obtain a theoretical concentration in the calibration range. The reference diltiazem $2 \%$ water solution was also analyzed after proper dilution, only with distilled water.

5. Analytical procedure - HPLC analysis - the stability tests were assayed by a Thermo Scientific UltiMate 3000 Analytical LC System, coupled with a Thermo Scientific Dionex UltiMate 3000 VWD-3100 Variable Wavelength Detector, following the procedure validated by Sadeghiet et al (3). The column - a Thermo scientific AQUASIL C18 (150 mm x 4.6 $\mathrm{mm}, 5 \mu \mathrm{m})$ and guard column - AQUASIL C18 $(10 \mathrm{~mm} \times 4.6 \mathrm{~mm}, 5 \mu \mathrm{m})$ - were settled at $50^{\circ} \mathrm{C}$. The test samples were introduced in a volume of $10 \mu \mathrm{L}$. The mobile phase (ethanol: aq. phosphoric acid with pH 2.2 - 35:65) was injected in an isocratic mode with a constant flow rate of $1.2 \mathrm{~mL} / \mathrm{min}$. The total run time was 8 minutes. The detector was set at $238 \mathrm{~nm}$. The peak areas were integrated automatically with the aid of a Thermo Scientific ${ }^{\mathrm{ru}}$ Chromeleon $^{\mathrm{nx}}$ 7.2 Chromatography Data System software.

\section{RESULTS AND DISCUSSION}

The chromatograms obtained with des-diltiazem standard solution $(100 \mu \mathrm{g} / \mathrm{mL})$ and diltiazem 75 $\mu \mathrm{g} / \mathrm{mL}$ : des-diltiazem $25 \mu \mathrm{g} / \mathrm{mL}$ mixture are shown on Fig. 2. Retention times for des-diltiazem and diltiazem were found at $2.6 \mathrm{~min}$ and $3.3 \mathrm{~min}$, respectively. Standard curves for both compounds, as described above, were obtained in the concentration range of $10-100 \mu \mathrm{g} / \mathrm{mL}$ (Fig. 3). 
Hydrolytic Stability of Diltiazem in the Presence of Chosen Polymers - Poloxamer 407 and Ammonio Metacrylate Copolymer Type B...
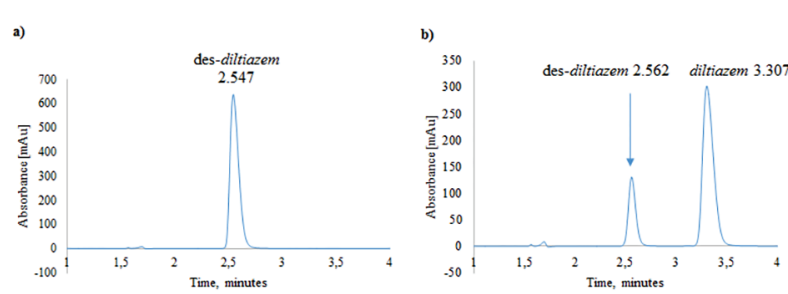

Fig. 2. Chromatograms of a) des-diltiazem standard solution $100 \mu \mathrm{g} / \mathrm{L}$ and b) diltiazem $75 \mu \mathrm{g} / \mathrm{mL}$ : des-diltiazem $25 \mu \mathrm{g} / \mathrm{ml}$ mixture

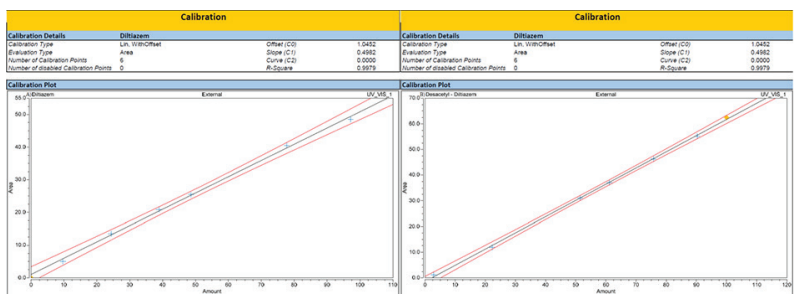

Fig. 3. Standard calibration curves of a) diltiazem and $b$ ) des-diltiazem

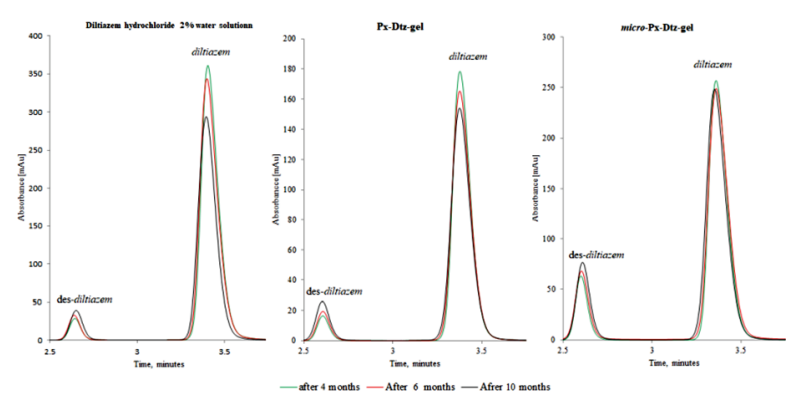

Fig. 4. Chromatograms of test formulations obtained at $4^{\text {th }}, 6^{\text {th }}$ and $10^{\text {th }}$ month of storing

The observed test samples showed substantially different degradation rates of diltiazem, decreasing in the order Poloxamer 20\%/ Eudragit RS-based hydrogel (micro-Px-Dtz-gel)> Poloxamer 20\% hydrogel (Px-Dtz-gel)>water solution. Fig. 4 presents the chromatograms obtained for each diltiazem dispersion, following the decrease in the parent drug peak area and the increase in the degradation product - desdiltiazem - peak area with the time. Under the studied conditions, no other degradation products of diltiazem were detected.

The change in the des-diltiazem content as a function of time for each dispersion is shown graphically on Fig. 5a. When diltiazem was introduced in a Poloxamer gel, it showed faster initial decomposition rate compared to its aqueous solution. Knowing the relatively great chemical inertness of the hydrogel polymer (Fig. 6a), such escalation of the hydrolysis process could be only explained with the shift in the dielectric conductivity of the media. Further decomposition acceleration was observed when the drug was included in the hydrogel via Eudragit $R S$ 100 sustained-release microparticles. Eudragit RS, also known with its pharmacopoeial name ammonio methacrylate copolymer Type B, contains 4.5 to 7.0 per cent quaternary ammonia 2-(trimethylammonio) ethyl 2-methylpropenoate groups (Fig. 6b) (14). Being positively charged, Eudragit $R S$ could be perceived as potential catalyst of the diltiazem hydrolytic degradation (1). The net negative effect on diltiazem stability was demonstrated to be only initial. After the $4^{\text {th }}$ month of storage, a linear steady-state increase in des-diltiazem concentration was reached. The kinet-
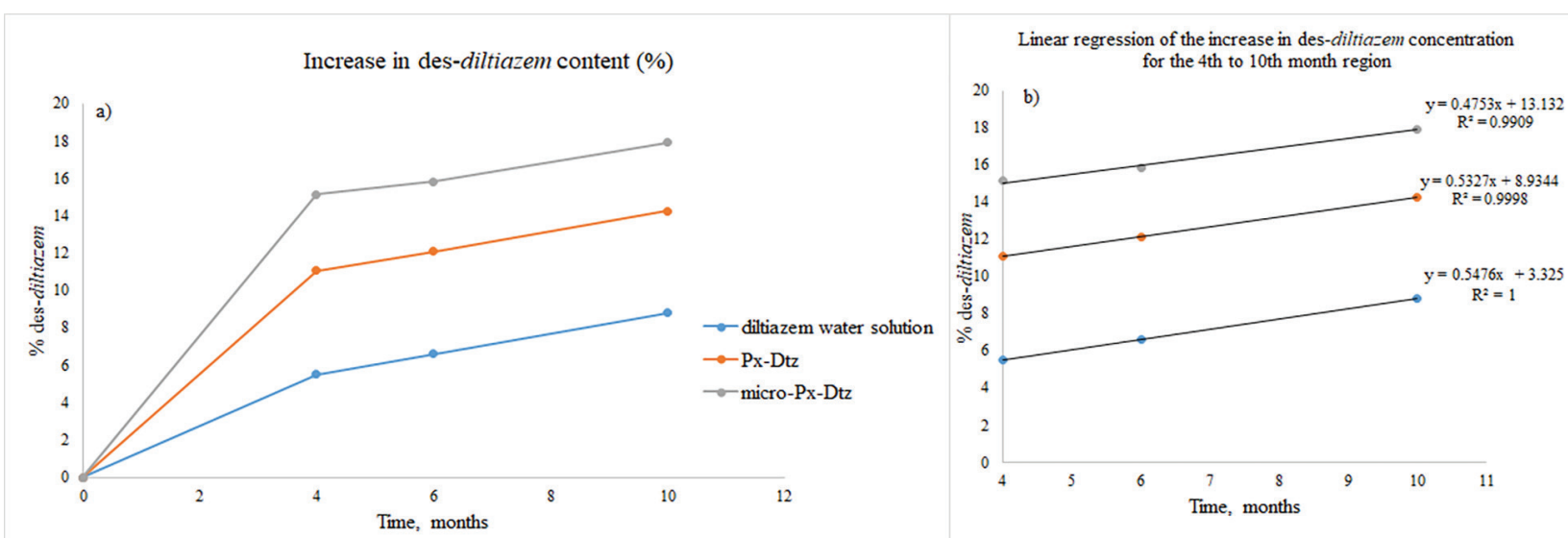

Fig. 5. a) Increase in des-diltiazem concentration (\% degradation) with time in test formulations; $b$ ) linear regression of the hydrolytic degradation using the data between the $4^{\text {th }}$ and the $10^{\text {th }}$ month of the study 
ic constant $(\mathrm{k})$ of the process is lowest for the microPx-Dtz-gel formulation ( $0.48 \%$ increase in des-diltiazem concentration per month) and highest for the aqueous solution $(0.55 \% /$ month) at this stage (Figure $6 \mathrm{~b})$. The observed phenomena is likely to be a result of a prevail of a viscosity based stabilization and a polymer "shield" determined stabilization after the $4^{\text {th }}$ month.<smiles>CC(O)COC(C)(C)COC(C)(C)CC(C)(C)OC(C)C</smiles><smiles>CCOC(=O)C(C)(CC(C)(C)C(=O)OC[N+](C)(C)Cl)C(=O)OC</smiles>

Fig. 6. Chemical structure of a) Poloxamer 407 and b) Eudragit $R S$

\section{CONCLUSION}

The complex kinetics of diltiazem hydrolysis in Poloxamer 407 gels suggest the presence of more than one factor with impact on the process. Usually, when this is the case, the concentration of the hydrolytic product increases in a polymodal manner, which turns the mathematical extrapolation of the experimental data in a complicated task. In our case, the polymodality of the des-diltiazem curves could be explained with the role of the polymer ingredients in the systems. The polymers - Poloxamer 407 and Eudragit RS - play a role of both - drug carriers and participants in the phase distribution, transition and dispersity of diltiazem. Hence, their physicochemical properties (crystallinity, stability, solubility, gelation, cross-linkage, etc.) and affinity to the drug molecule will be the factors determining the rate and order of diltiazem hydrolysis. Again, the intermolecular catalysis is not excluded, especially in the case of $\mathrm{Eu}$ dragit $R S$-enriched gels.

Despite the lack of complete straightforwardness in the results, the main conclusions to be drawn are:

1. Diltiazem possesses complex decomposition kinetics in Poloxamer 407 hydrogels (with or without Eudragit RS microsponges); likely due to the influence of multiple factors on the hydrolysis process, related to the impact of drug nature and concentration, polymer participation and phase distribution.

2. The enhanced viscosity of hydrogels was not found to benefit the hydrolysis stability of diltiazem.

3. The incorporation of diltiazem in a sustained-release microsponge drug delivery system composed by Eudragit $R S$ did not demonstrate the expected protection effect against the environmental hydrates. This observation was explained with the possible catalytic effect of cationic Eudragit $R S$ centers on the hydrolysis of diltiazem.

\section{REFERENCES}

1. Yoshioka S, Stella VJ. Stability of drugs and dosage forms. Kluwer Academic Publishers; 2002. doi: 10.1007/0-306-46829-8_4.

2. Chafetz L, Shah KP. Stability of diltiazem in acid solution. J Pharm Sci. 1991;80(2):171-2. doi: 10.1002/jps.2600800218.

3. Sadeghi F, Navidpour L, Bayat S, Afshar M. Validation and uncertainty estimation of an ecofriendly and stability-indicating hplc method for determination of diltiazem in pharmaceutical preparations. J Anal Methods Chem. 2013;2013:1-10. doi: $10.1155 / 2013 / 353814$.

4. Mazzo DJ, Obetz CL, Shuster J. Diltiazem Hydrochloride. In: Brittain HG, editor. Analytical profiles of drug substances and excipients. Elsevier: Academic Press, Inc; 1994. p. 53-98. doi: 10.1016/ s0099-5428(08)60600-0.

5. Molden E, Johansen PW, Boe GH, Bergan S, Christensen H, Rugstad HE, et al. Pharmacokinetics of diltiazem and its metabolites in relation to CYP2D6 genotype. Clin Pharmacol Ther. 2002;72(3):333-42. doi: 10.1067/mcp.2002.127396. 
Hydrolytic Stability of Diltiazem in the Presence of Chosen Polymers - Poloxamer 407 and Ammonio Metacrylate Copolymer Type B...

6. Molden E. Desacetyl-diltiazem displays severalfold higher affinity to CYP2D6 compared with CYP3A4. Drug Metab Dispos. 2002;30(1):1-3. doi: 10.1124/dmd.30.1.1.

7. Muszalska I, Jamszol L, Grzeskowiak D. Kinetics of hydrolysis of diltiazem hydrochloride in aqueous solutions. Acta Pol Pharm. 2003;60(3):163-168. doi: 10.1111/j.1365-2710.1988.tb00215.x.

8. Ishii K, Minato K, Nakai H, Sato T. Simultaneous assay of four stereoisomers of diltiazem hydrochloride. Application to in vitro chiral inversion studies. Chromatographia. 1995;41(7-8):450-4. doi: 10.1007/bf02318621.

9. Ishii K, Wakamoto S, Nakai H, Sato T. Assay of four stereoisomers of desacetyl diltiazem hydrochloride. Application to in vitro chiral inversion studies. Chromatographia. 1996;43(7-8):413-8. doi: 10.1007/bf02271021.

10. Molden E, Christensen H, Sund RB. Extensive metabolism of diltiazem and P-Glycoprotein-mediated efflux of desacetyl-diltiazem (M1) by rat jejunum in vitro. Drug Metab Dispos. 1999; 28(2):107109. doi: 10.1124/dmd.30.1.1.
11. Ivanova NA, Trapani A, Franco CD, Mandracchia D, Trapani G, Franchini C, et al. In vitro and ex vivo studies on diltiazem hydrochloride-loaded microsponges in rectal gels for chronic anal fissures treatment. Int J Pharm. 2019; 557:53-65. doi: 10.1016/j.ijpharm.2018.12.039.

12. Kolev IN, Ivanova NA, Marinov MK, Alexieva GE, Strashilov VL. A QCM-based assay of drug content in Eudragit RS 100-based delivery systems. Talanta. 2019;202:531-9. doi: 10.1016/j.talanta.2019.05.033.

13. Ivanova NA, Franco CD, Trapani A, Petkova V, Dimitrov M. Synthesis and characterization of diltiazem loaded Eudragit RS 100 "microsponges". World J Pharm Pharm Sci. 2017;6(6):32-42. doi: 10.20959/wjpps20176-9275.

14. Council of Europe. European Pharmacopoeia 8th ed. Strasbourg: Council of Europe; Ammonio Methacrylate Copolymer Type B. 2008;1551-52. 